\title{
A new Zoning Graded Non-uniform Grid Generation Algorithm Including the Equivalent Width of Technology
}

\author{
Feixiang Gong, Bo Yin, Yanping Cong, Qing Yan, Haokun Chi \\ Ocean University of China, Qingdao, Shandong, 266100, China \\ gongfeixiang@gmail.com
}

Keywords: geometric non-uniform grids; FDTD

\begin{abstract}
Analysis of the generation principle finite difference time domain (FDTD) grid, proposed non-uniform meshing algorithm based on the equivalent wi-dth of the geometric. In meshing software, we set $\mathrm{R}_{\mathrm{x}}, \mathrm{R}_{\mathrm{y}}, \mathrm{R}_{\mathrm{z}}$. If reduced size along the $\mathrm{x}$-axis model, it can be coarse mesh point on the basis of compliance with the numerical dispersion equation. Due to in the square mesh meshing size in the $\mathrm{x}, \mathrm{y}, \mathrm{z}$ direction is constantly changing,so get the equivalent size of the actual model, you need to obtain the average of the grid cell size along the direction of the coarse grid, is the ratio of the number of grid and size of the model. The half-size model of the grid is reduced by this average in the corresponding direction and getting the equivalent size model. And repeat the first step, conducted mesh. By numerical verification prove the accuracy and effectiveness of the new zoning graded non-uniform grid generation algorithm including the equivalent width of Technology in the FDTD simulation.
\end{abstract}

\section{Introduction}

When using the finite difference time domain (Finite Difference Time Do-main, FDTD) method for the calculation of electromagnetic field numerical model , the model must first be considered subdivision . Mesh is good or bad, not only affects the accuracy of the results,but also the degree of convergence of computing processes, and thus a good mesh for the entire FDTD algorithm must be calculated of great significance. Description of the grid should be consistent with changes in the electromagnetic field . If the calculation domain both use fine mesh to split, when it run ,it is bound to take up too much of the computer resources and a longer running time ; If the calculation domain both use coarse mesh to split, it will give the FDTD calculations bring large errors. To solve this problem, we usually use non-uniform FDTD method ${ }^{[1-6]}$. Literature ${ }^{[4]}$ introduces approach that mesh size in the space to make certain rules extended by non-uniform grid method. The basic idea is to make the axial mesh density increases by geometric progression . Because the entire grid space is get geometric progression increasing ,we can obtained manner such that the field component 6 iterative good consistency by This mesh generation method. Li Xiang et al ${ }^{[2]}$ on the basis of the expansion of the grid algorithm improves the extended partition mesh algorithms and algorithms used in the non-uniform grid partition graded in its preparation of non- uniform partition software and set and achieve cross-section visualization of the results. Zhou Guoxiang et al ${ }^{[3]}$ also visually realized an universal and reasonable non-uniform mesh algorithm, made more reasonable non-uniform meshing algorithm is a very versatile tool that combines coarse mesh partition graded non-uniform grid algorithm. Non-uniform mesh with the general method of partitioning graded compared to non-uniform grids have been greatly improved, but there are some limitations : The minimum mesh size and the constant of proportionality to be artificial ; in process algorithm processing, essentially by adjusting the minimum mesh size of the grid correspond to the proportion of each $\Delta \mathrm{x}_{\min } \mathrm{Rx}$ so that it can meet the requirements, the result of this process is not only poor control and may produce some unexpected results ${ }^{[7]}$. for how $\Delta \mathrm{x}_{\min }$ and make the appropriate adjustments to get more satisfactory results, the literature does not give a reasonable approach. Based on the basic idea of this algorithm, we propose a non-uniform meshing algorithm based on geometric equivalent width . 


\section{Improved algorithm for geometric non-uniform mesh}

Geometric non-uniform meshing algorithms to solve the region along the $\mathrm{x}, \mathrm{y}, \mathrm{z}$ direction is divided into multiple sub- space to $\mathrm{x}$ direction , for example, set up the initial grid cell is the smallest $\Delta \mathrm{xmin}$, $\mathrm{x}$ direction ratio $\mathrm{Rx}(\mathrm{Rx} \in[1,1.25])$. After meshing, $\Delta \mathrm{l}$ subspace dense to sparse and then showing dense form .

The equivalent width of the simulation model is a certain width correction, so that when the solution space using the coarse mesh, fine mesh can be obtained as the same accurate simulation results $^{[8]}$. Total number of grid cells to reduce the use of this method in reducing the amount of computation time and computer memory while still able to get accurate results. Here 's the equivalent of about half the size than the actual size of a small grid cell size.

In this paper, the equivalent geometric algorithms and non-uniform width of the algorithms proposed by combining the equivalent width based on non-uniform algorithm for solving the following steps:

Set in meshing software $\Delta x \min , \Delta y \min , \Delta$ zmin and $\mathrm{Rx}, \mathrm{Ry}, \mathrm{Rz}$, if miniature size along the $\mathrm{x}$ -axis , $\Delta x \min$ can meet the numerical dispersion large set of formulas on the basis of a little coarse mesh .

Due to the size of the non-uniform mesh in the square $\mathrm{x}, \mathrm{y}, \mathrm{z}$ direction is constantly changing, so when you get the equivalent in size of the actual model, you need to seek the direction of the coarse grid is divided into a grid cell size and it means that the size of the model in the direction of the ratio of the number of grids will reduce the size of half a calculation model in the corresponding direction through the grid this average to obtain the size of the equivalent model ${ }^{[9]}$.

Repeat the first step , conducted mesh .

\section{Experimental verification}

According to the above algorithm, application of electromagnetic simulation software HFSS and geometric non-uniform meshing software for a microstrip patch antenna triangulation and electromagnetic analysis, the results shown in Fig.1-4.

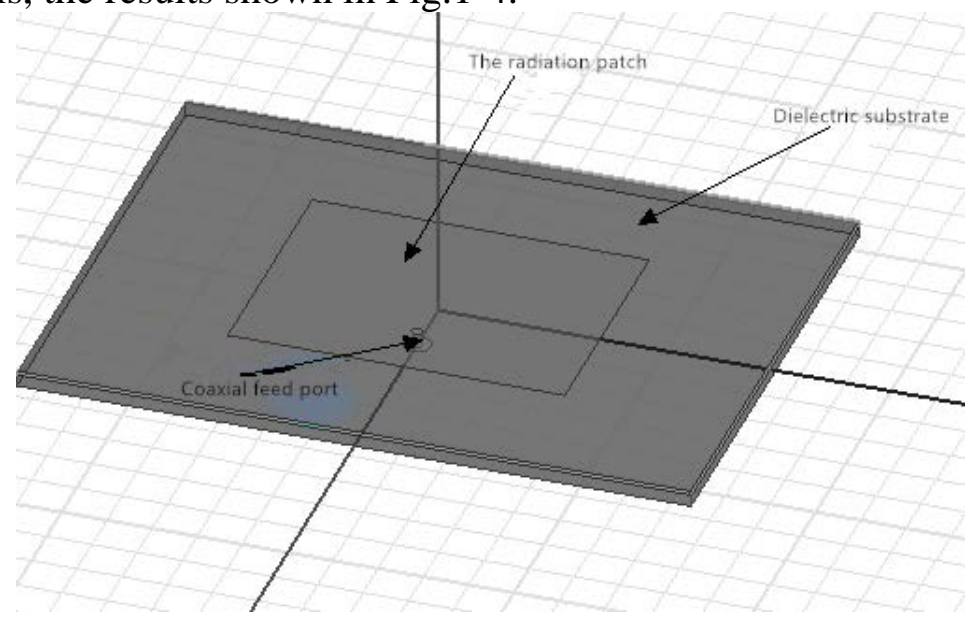

Fig.1coaxial fed rectangular microstrip antenna design model

After the designing for simulation model, we use fine mesh generation software to split mesh, and set values : $\mathrm{Rx}=1.1, \mathrm{Ry}=1.1, \mathrm{Rz}=1.1$. And then put the result into HFSS to make simulation analysis. After the simulation is complete, we determine the calculated time of 5 minutes and 41 seconds, and check the antenna sweep analysis $1.5 \sim 3.5 \mathrm{GHz}$ band, as well as absorbing boundary electric field distribution of the magnetic field distribution inside the microstrip antenna, as shown in Fig.2. 


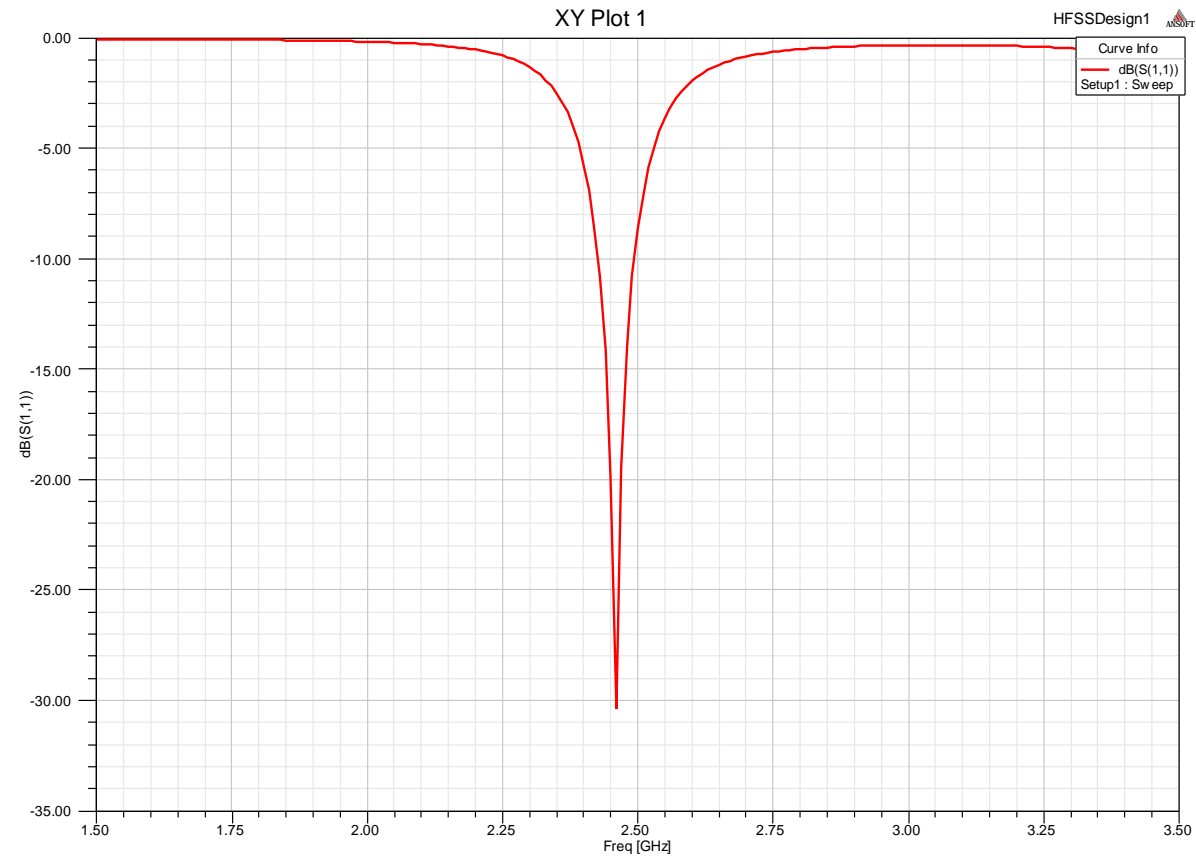

Fig. 2s11 sweep analysis

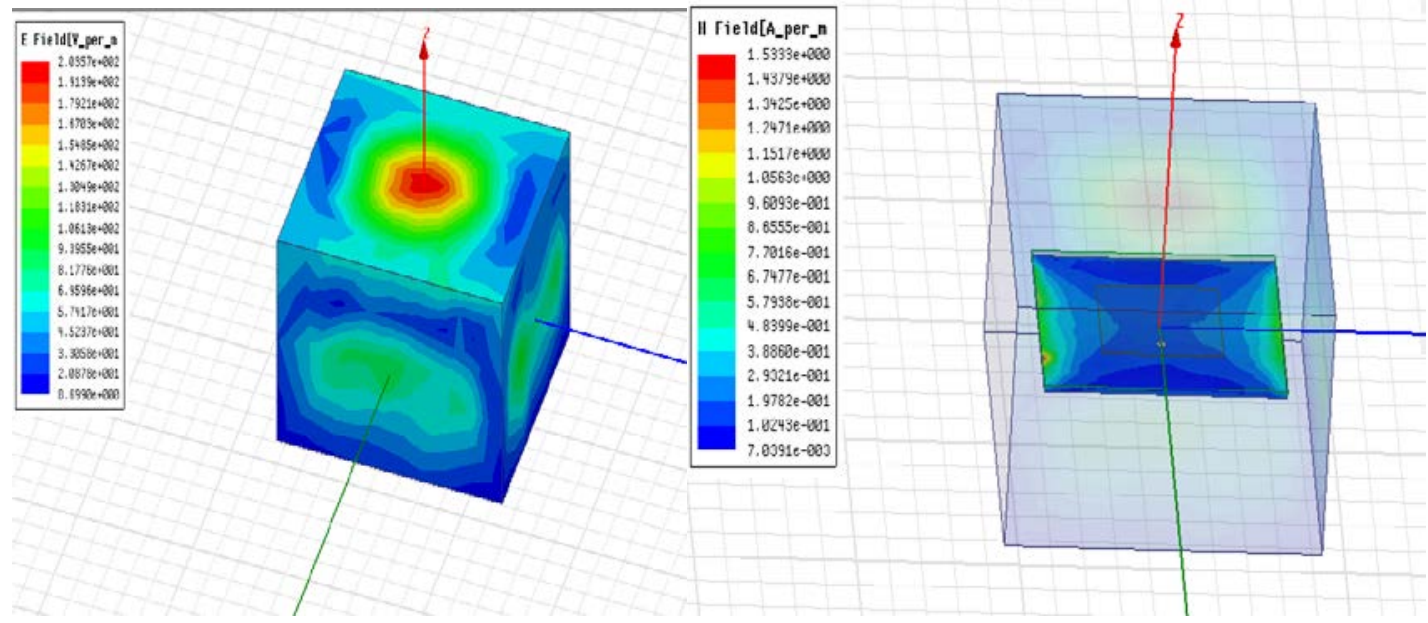

Fig.3absorbing boundary and the electric field distribution of the magnetic field distribution inside the microstrip antenna

Coarse mesh with $\mathrm{Rx}=1.1, \mathrm{Ry}=1.1, \mathrm{Rz}=1.1$ also put into HFSS to make simulation analysis to determine the results of the calculation time is swept 3 minutes and 20 seconds after the analysis is complete, the electric field distribution and the magnetic field inside the microstrip antenna absorbing boundary distribution, as shown in Fig.4-5. 


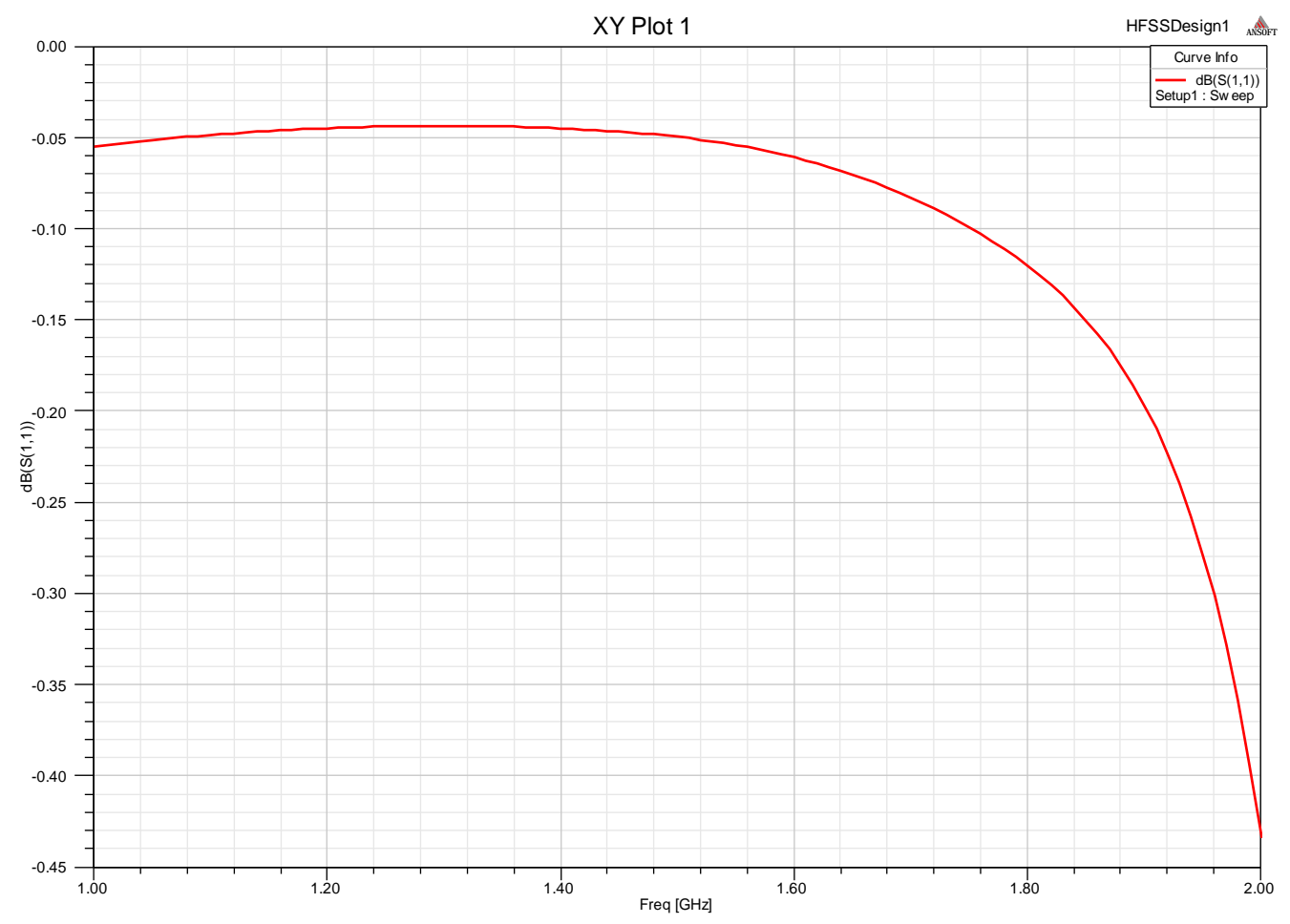

Fig. 4s11 sweep analysis

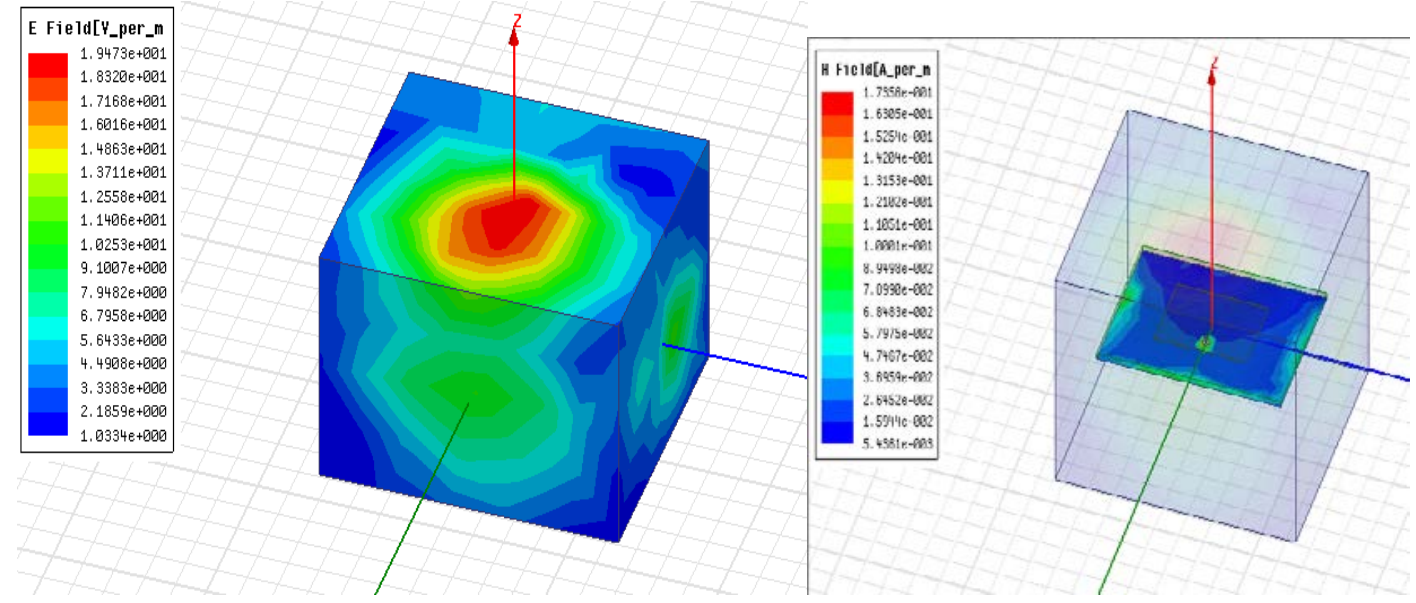

Fig.5 absorbing boundary and the electric field distribution of the magnetic field distribution inside the microstrip antenna

From the analysis of the results of the two methods triangulation sweep analysis results, the magnetic field distribution of the electric field distribution as well as absorbing boundary microstrip antenna inside is totally different, there is a big gap between the electromagnetic field size.

The document shows the results of the query mesh, while coarse mesh grid cells 440 become symbiotic, the study was divided into a grid space of $11 \times 20 \times 2$, the $x$-axis direction 11 , so according microstrip antenna along the $x$-axis length $\mathrm{L}$ is $28 \mathrm{~mm}$, so the average grid cell size of $2.54 \mathrm{~mm}$, the length minus $1.27 \mathrm{~mm}$, continue to be the coarse mesh grid, and the results of HFSS simulation analysis, the analysis is complete after determining the computation time of 3 minutes 52 seconds, sweep analysis results, as well as absorbing boundary microstrip antenna electric field distribution inside the magnetic field distribution, as shown in Fig.6-7: 


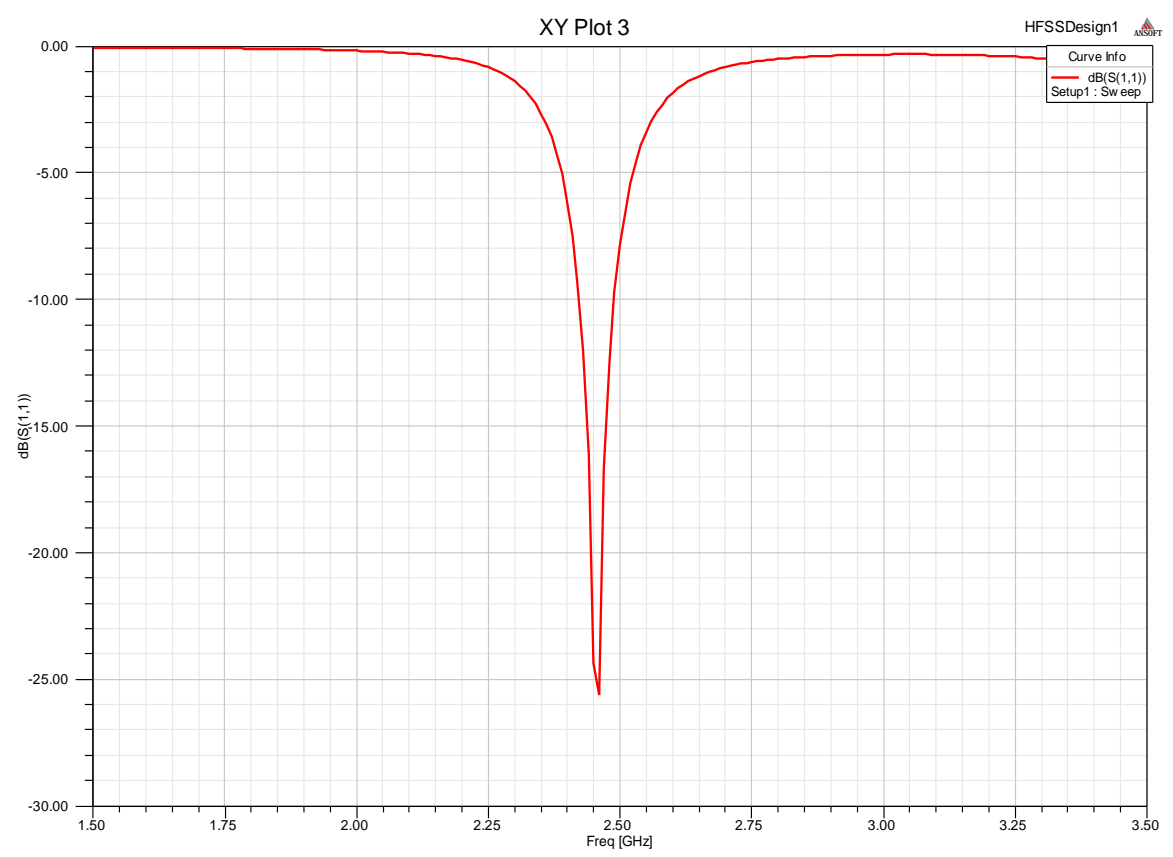

Fig. 6 s11 sweep analysis

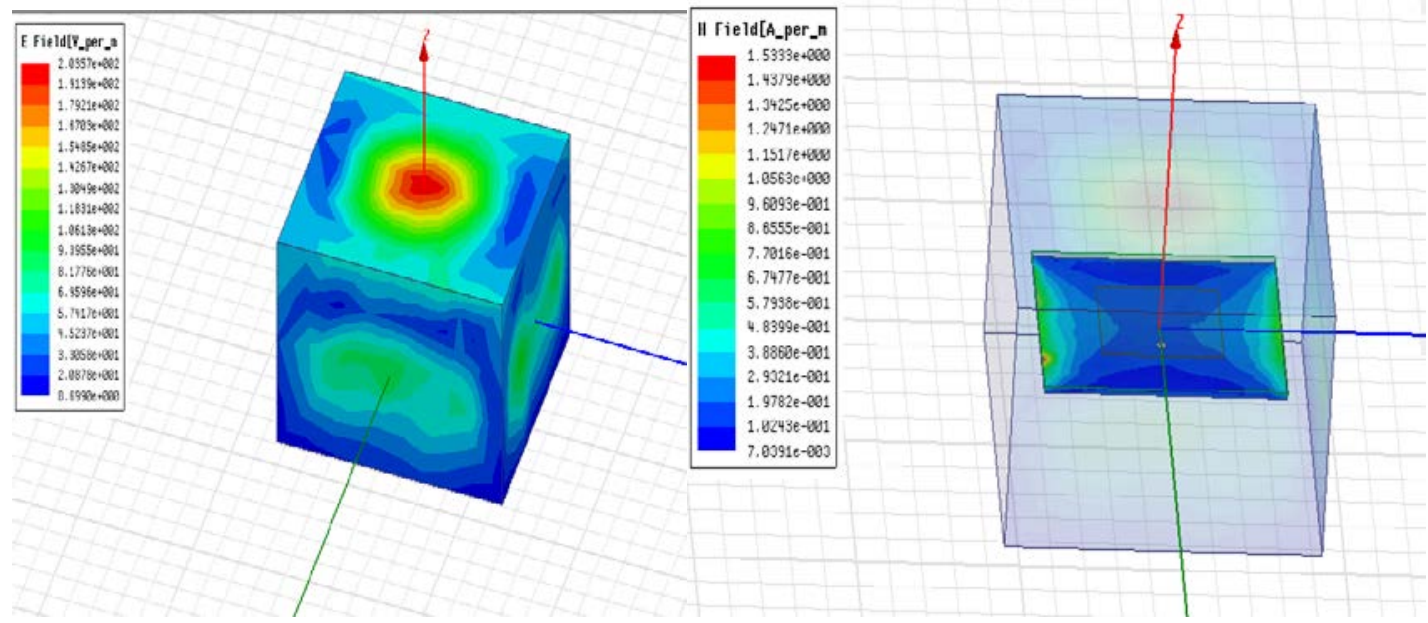

Fig. 7 absorbing boundary and the electric field distribution of the magnetic field distribution inside the microstrip antenna

As can be seen from the simulation results, the use of improved algorithm sweep analysis results $^{[10]}$, as well as absorbing boundary of the magnetic field distribution of the electric field distribution inside the microstrip antenna is basically the same, so verify the rationality of this algorithm.

\section{Conclusion}

This paper presents a geometric based on the equivalent width of the non-uniform mesh generation algorithm. The improved algorithm have solved the space division compared with other algorithms and this algorithm join the equivalent width of the technology, so the cross-section through a coarse grid accurate results can be obtained a fine mesh FDTD calculation time ${ }^{[11]}$. The minimum mesh size can be set on the basis of the dispersion and does not occur on a large number, so the algorithm use more less generated mesh compare with other algorithms on the total number of grid cells ${ }^{[12]}$. Furthermore, the algorithm is incremented by a geometric grid cell size and associated with the largest grid, so grid cells can flatly increase when making the solution space, and it can be avoid the phenomenon that the difference of adjacent mesh size is too large to occur numerical dispersion. In a short, this meshing algorithm generates fewer number of grids, greatly reducing the computation time and improve the accuracy of the calculated results. 


\section{Acknowledgement}

In this paper, the research was sponsored by the Human implantable electronic devices NMR wireless Transmission power system joint research (Project No. 2013DFA10490) and Qingdao strategic industry development project (Project No. 13-4-1-15-HY) and Marine comprehensive information three-dimensional monitoring network key technology based on software radio (Project No. 2013GHY11519).

\section{References:}

[1]GAO Ben-qing. Finite-Difference Time-Domain Method [M].Beijing:National Defense Industry Press,1995.(in Chinese)

[2]LI Xiang, YANGMing-wu, WANG Wei-ji,et al.Study of FDTDElectromagnetismModeling System[J].Information and Elec-tronic Eegineering,2005,3(4):257-260.(in Chinese)

[3] ZHOU Guo-xiang, CHENG Ping, JIANG Jing-guo, et al. A Non-uniform FDTD Mesh Generation System in Cartesian Co-ordinate[J]. Journal of Microwaves, 2005,21(2): 56-59.(in Chinese)

[4]YAOGuang-feng,WANG Ji-qin,LIU Gang. A Study on the PMLAbsorbing Boundary Condition of the FDTD Method for Rectangle Microstrip Antenna[J].Modern Radar, 2003, 25(11):36-38. (in Chinese)

[5]Reineix A,Jecko B.Analysis of Microstrip Patch Antennas Us-ingFinite Difference Time Domain Method[J].IEEE Transac-tions on Antennas and Propagation,1989,37(11):1361 -1369.

[6]David M Sheen, Sami MAli, Mohamed D Abouzahra,et al.Application of the Three-Dimensional Finite-Difference Time-Domain Method to the Analysis of Planar Microstrip Circuits[J]. IEEE Transactions on Microwave Theory and Tech-niques,1990,38(7):849-857

[7] V. Jandhyala, et al. A combined steepest descent-fast multipole algorithm for thefast analysis of three-dimensional scattering by rough surfaces. IEEE Trans. Geosci.Remote Sensing, 1998, 36(3): 738-748.

[8] C. D. Moss, et al. Forward-backward method with spectral acceleration forscattering from layered rough surfaces. IEEE Trans. Antennas and Propagat., 2006,54(3): 1006-1016.

[9] M. Y. Xia, et al. An efficient algorithm for electromagnetic scattering from roughsurfaces using a single integral equation and multilevel sparse-matrixcanonical-grid method. IEEE Trans. Antennas Propag., 2003, 51(6): 1142-1149.

[10] D.C.olak, R. J. Burkholder, and E. H. Newman. Multiple sweep method ofmoments analysis of electromagnetic scattering from 3D targets on ocean-likerough surfaces. Microwave and Opt. Tech. Lett., 2007, 49(1): 241-247.

[11] M. El-Shenawee. Polarimetric scattering from two-layered two-dimensionalrandom rough surfaces with and without buried objects. IEEE Trans. Geosci.Remote Sensing, 2004, 42(1): 67-76.

[12] S. Benkler, N.Chavannes, and N. Kuster. A new 3-D conformal PEC FDTDscheme with user-defined geometric precision and derived stability criterion. IEEETrans. Antennas Propag., 2006, 54(6): 1843-1849. 